\title{
Modeling of an Actif Cyclic Electromagnetic Effect on Hemozoin for Malaria Diagnosis Computerized Electromagnetic Effect on Hemozoin for Malaria Diagnosis
}

\author{
Walvede Biziya Deli, Ntsama Eloundou Pascal, Talla Emmanuel, Kuitche Alexis \\ University of Ngaoundere, Electrical and Electronics Engineering Laboratory, Ngaoundere, Cameroon \\ Email address: \\ Walvedebiziya@gmail.com (W. B. Deli) \\ To cite this article: \\ Walvede Biziya Deli, Ntsama Eloundou Pascal, Talla Emmanuel, Kuitche Alexis. Modeling of an Actif Cyclic Electromagnetic Effect on \\ Hemozoin for Malaria Diagnosis Computerized Electromagnetic Effect on Hemozoin for Malaria Diagnosis. American Journal of \\ Electromagnetics and Applications. Vol. 6, No. 1, 2018, pp. 28-34. doi: 10.11648/j.ajea.20180601.15
}

Received: May 14, 2018; Accepted: June 21, 2018; Published: July 17, 2018

\begin{abstract}
In this work we present the heat generated by the super magnetic particle, the waste prevent from the plasmodium degradation. The hemozoin behaviour due to electromagnetic field applied on it will be used as a sensitive method for malaria diagnosis. Hemozoin presents its self in the form of a crystal and which constitutes a pigment synthesised by the malaria parasite with the aim of annihilating the toxic effect ofhematine. In effect, after contamination the plasmodium dwells inside the red blood cells of the host so as to feed itself with the amino-acids obtained by degradation of hemoglobin. But during this "meal" the parasite liberates a waste product in the form of a complex made of porphyrin and iron. In order to prevent that noxious effect, the plasmodium polymerizes the complexes of porphyrin inside a digestive vacuole to finally produce a crystal of a hemozoin not toxic for him. This particle is previously paramagnetic because of the presence of iron in the red blood cells. This paper proposes the modeling of magnetic field effect of hemozoin for malaria detection. The potential effect of the cyclically activated magnetic field was modeled using a thermodynamic framework. Then at the end, generation and diffusion of heat due to the presence of constant cyclically magnetic field have been determined. These to prove how the pull or push forces between the particles of Hemozoin are demonstrated by producing the heat. The energy inside the particle is also taken into consideration in the cell while doing the diagnosis of malaria.
\end{abstract}

Keywords: Modeling, Electromagnetism, Hemozoin, Diagnosis, Malaria

\section{Introduction}

Hemozoin is a waste product of the malarial parasitic action on hemoglobin. Thus, its presence in the blood of an individual means the presence of the disease in that organism. It has been proven that even not the indigenes do acquire immunity against malaria, so that without diagnosis, infection still occurs, and may lead to dead [1]. The worldwide eradication trials of malaria have always failed because of the vector's resistance to insecticides and that of the parasite towards anti-malarial drugs. Actually, the reference technique, considered as best method for the diagnosis of malaria is microscopy [2]. This diagnosis technique requires a specialized laboratory with qualified microscopists; and may cause hematomas due to blood collection. Additionally, the laboratory equipment (microscopes) required to perform microscopy is really expensive for the tropical dwellers, who constitutes the mostly affected population. Taking into consideration all thoses limits of this technique, it is important to come out with new malaria detection methods, which shall be as reliable as the actually approved methods; while extending their limits. It is in this sense that we made an attempt in exploiting the magnetic properties of Hemozoin in order to detect malaria parasites in the blood using the susceptibilities as main parameters of electromagnetic Lab. Test of hemozoin in an infected blood due to plasmodium fp presence. In the first section the hemozoin in human cell will be idealized as magnetic nanoparticles that are embedded in an infected blood. In the second section, we present the potential effect of the cyclically activated magnetic field modeling using a thermodynamic framework; and finally, the results and the 
conclusion are presented in the last section.

\section{Hemozoin}

The Malaria parasite generates a super magnetic particle, known as hemozoin, during the process of feeding [1]. Hemozoin is the disposal product formed from the digestion of blood feeding parasite. It is formed within the acidic aqueous ( $\mathrm{pH} 4.8$ ) environment of the digestive food vacuole of the plasmodium falciparum [2]. Since the formation of the hemozoin is essential for the survival of these parasites, it is therefore an attractive target for developing drugs and is much studied in plasmodium as a way to find drugs to treat malaria. The hemozoin crystal is about 100-200 nm long and each contains about 80,000 heme molecule [3].

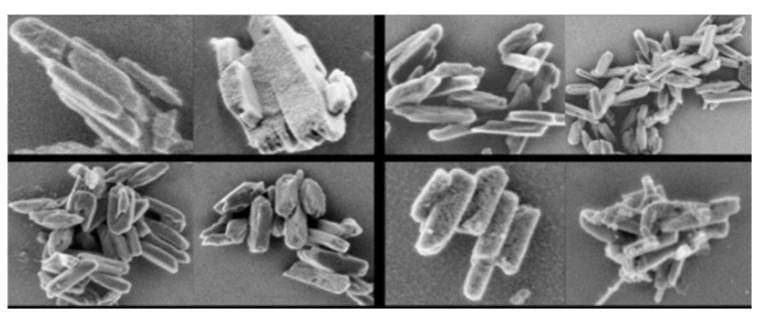

Figure 1. SEM images of hemozoin.

\subsection{Oscillations of the Hemozoin}

Oscillation is the repetitive variation between two or more different states. Oscillation occurs both in physics system and biological system [4]. When a mass is attached to a linear spring, subjected to no external force, such a system is said to be in equilibrium when the spring is static. However, when the system is displayed, there is a net restoring force which tends to bring it back to equilibrium, in this process, the mass acquire a momentum [5]. Current research in this field indicates that oscillating magnetic field could cause oscillation of the hemozoin, leading to a Mechanical damage of the organelles of the parasite. Mechanical stresses are known to cause cell death via apoptosis [6].

\subsection{Hysteresis}

Hysteresis systems are path dependence, the output depends in part on the internal state of the system and not only on its input [7]. If an alternating magnetic field is applied to a material, its magnetization will trace out a loop called hysteresis loop [7]. Therefore a hysteresis loop shows the relationship between the induced magnetic flux density and the magnetizing force [8].

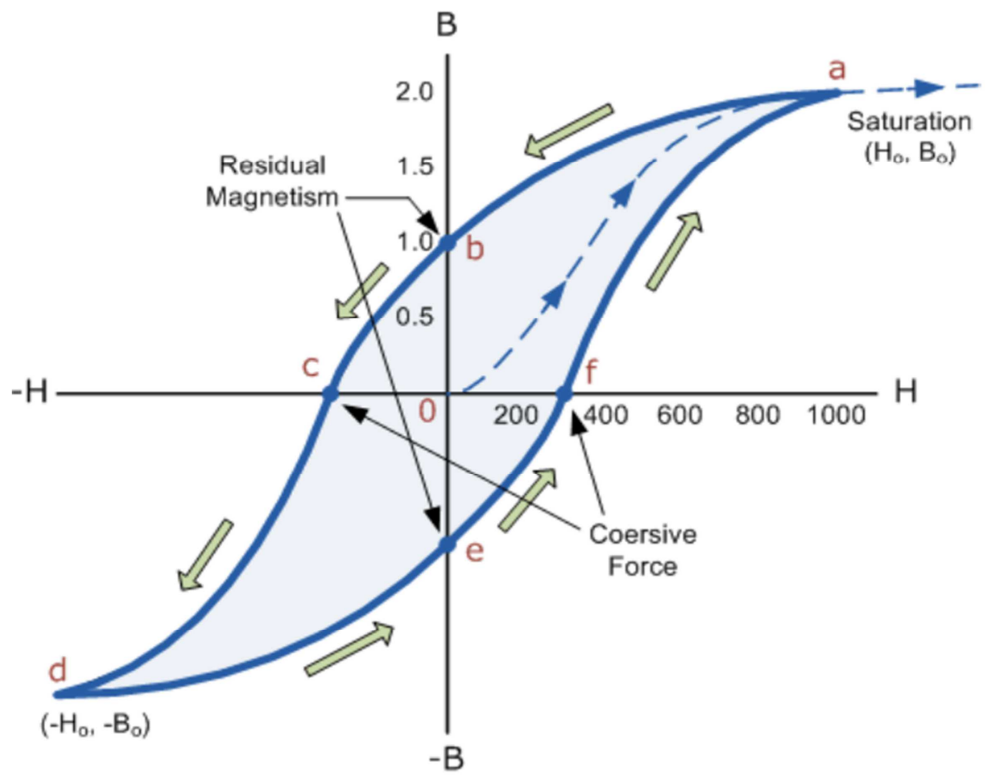

Figure 2. Magnetic hysteresis loop.

By studying the hysteresis loop, much information on the magnetic property of a material can be understood. This leads to the concept of constant flow of energy into that material when you apply a time varying magnetic field to that ferromagnetic material. This is the physics basis of hyperthermia treatment [9]. The shape of the loop is partly determined by the particle size. Particles at the nano-scale exhibits superparamagnetism [9]. When an external magnetic field is applied to a nano-particle, the magnetic moments align alongthe applied field.

\subsection{Magnetic Field}

Magnetism is defined as the force produced by charge particles of a magnet. Every charged particle produced a magnetic field when it moves. For instance, the spinning of electrons in an atom generates a magnetic field around it. The magnetic Field strength of an electromagnet depends on the number of the turns of the coil and the current flowing through the coil as well as the type of material used. Magnetic fields are used as external stimuli to control the behaviour of complex geometry of magnetic nanoparticles 
[11]. By moving a wire or changing a magnetic field, there is an induced voltage and current within the coil. This process is called electromagnetic induction [12].

\subsection{Magnetic Materials}

Magnetic materials under the influence of external magnetic field show different characteristics. There are soft magnetic material and hard magnetic material
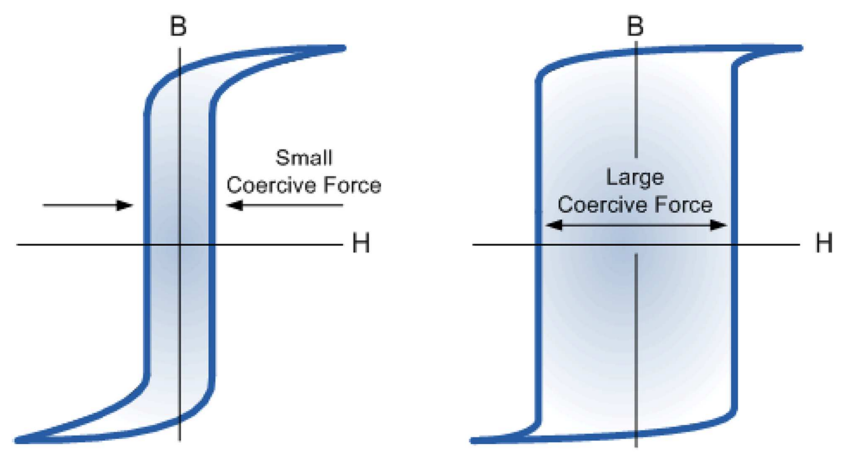

Figure 3. Soft and Hard magnetic material.

Their response to the field is dependent upon temperature. When an external magnetic field is applied to a material, the magnetic moment of the electrons aligns themselves with the $\mathrm{B}$ so that the net magnetic field is not zero. The paramagnetic material is displayed by substances that have unpaired electrons in their atomic orbital. They have non-interacting spins that is characterized by linear susceptibility which is inversely dependent on the Curie temperature [13]. The ferromagnetic materials are characterized by exchange interactions, and hysteresis. They are composed of magnetic domains which points in random direction leading to a zero net magnetic moment. They retain their magnetic moment after the external filed is switched doff. By continuous reducing the size of the materials, the magnetization measured becomes zero, thus there is no coercively in such a material and therefore, it behaves like a paramagnetic or super paramagnetic nanoparticle.

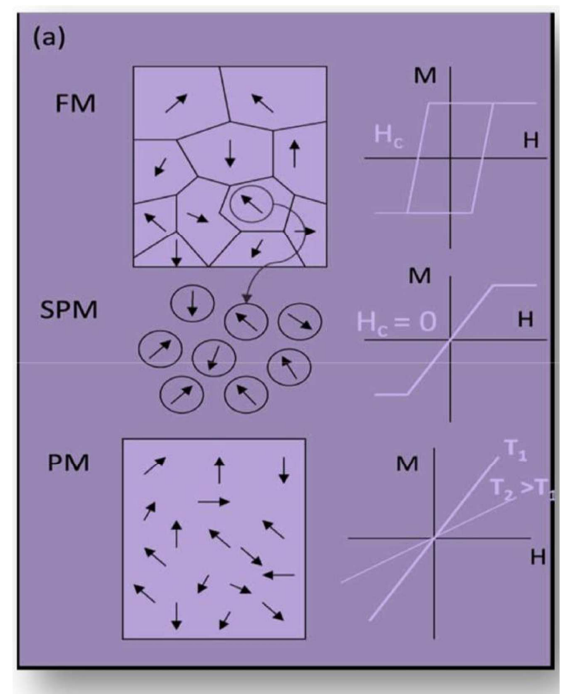

Figure 4. Magnetic materials showing different magnetic behavior.

\subsection{Effect of Heat on Magnetic Nanoparticle}

When magnetic nanoparticles are subjected to electric current, the current causes the MNPs to spin about their moment which eventually lead to their death. These magnetic nanoparticles possess super paramagnetic property as a result of exposure to AC [11]. Magnetic materials under the influence of $\mathrm{AC}$ are convenient for hyperthermia application [10]. Rosensweig gave detailed information based on Debye model for dielectric dispersion in polar fluids on the heating process for the magnetic nanoparticle. Application of an alternating magnetic field to a magnetic nanoparticle makes the magnetic moment of the MNP to rotate and align with the changing filed.

\subsection{Cyclic Electromagnetic Field}

One such advantage of using cyclic electromagnetic filed is that the field increases the sensitivity of the parasite to low concentration of anti-malarial drugs and, therefore enhances the effective treatment of the disease [14]. The oscillating magnetic field may affect the parasites in two ways, according to Lai. In organisms still in the process of binding free heme molecules into a large number, the field causes the stacked heme molecules to shake thus preventing more stacking. The oscillating field also causes the stacks to spin, which cause damage and death of the parasite [14].

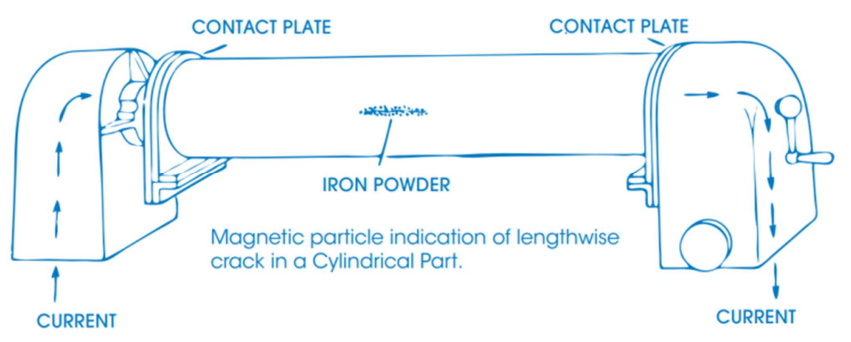

Figure 5. Oscillating field for iron powder detection.

Systems undergoing resonance are often used to generate vibrations at specific frequencies. As the parasites oscillate there is also an effect that tends to reduce the amplitude of the oscillation. This effect is known as damping, which is as a result of some losses from cycle to cycle. This oscillation causes vibrations in all direction between the parasites which effectively result in collision and eventually leads to the generation of heat. As the particles collide with each other, they gain momentum and are able to travel past their mean position [15].

\section{Heat Generation}

\subsection{Effect of Oxillating Magnetic Field}

When an oscillating magnetic field is applied to the malaria parasite, the oscillation of the field produces the heat generation. The heat generation is due to the collision of the particles inside the hemozoin. Therefore, according to Lai, treating over 500 million people every year who are infected with malaria using oscillating magnetic field would be inexpensive and very simple [14]. In this research, we 
considered the particles inside the heme as a magnetic nanoparticle. The formation of the hemozoin prevents oxygen radical-medicated damage to the malaria parasite heat under oscillating fields by physical mechanisms such as relaxation loss or hysteresis loss. The oscillating field influences the heating of the nanoparticle. Magnetic nanoparticles generate these generally depend on the frequency of the field as well as the particle size biological systems at the molecular level [16]. Magnetic nanoparticles can be manipulated Recent research has shown that magnetic nanoparticles with size of 5-200 $\mathrm{nm}$ can induce heat in using a magnetic field. Their physical and chemical properties depend on their method of synthesis as well as their chemical structure [17]. In this research however, we focus on the ferrite nanoparticles because Ferrite nanoparticles exhibit magnetic behaviour once an external magnetic field is applied to it [18]. Therefore, we anticipate that considering the particles inside the hemozoin as magnetic nanoparticles, they can be trapped inside, as external field is applied causing an induced heat and eventually make it inactive. The size and shape determines the response of the nanoparticle to the induced heat [19]. The rate of heat dissipation of nanoparticle is inversely related to the particle size, hence small particle size should enhance the level of heating, and the thermodynamics of the heated particle can be modeled using the first law of thermodynamics.

\subsection{Temperature in Adiabatic System}

In order for us to determine the temperature, we decided to specify the temperature distribution along our system at an initial condition. Assuming an adiabatic system in which there is no heat transmitted through the surface and on the system, a finite difference scheme of forward, backward and central difference approximations was used to estimate the temperature gradients within the plane.

\section{Methods}

The physics-based approach is used to model the effects of cyclic activated magnetic fields on the localized heating of the malaria infected tissue in the female anopheles mosquitoes. The project will be carried out in the following stages:

The hemozoin in the parasites will be idealized as clusters of magnetic nanoparticle that are embedded in the tissue.

The potential effects of the cyclically activated magnetic field will then be modeled analytically within a thermodynamic framework

The heat generation and diffusion due to the cyclically activated field then be modelled within a computational framework that will be implemented using finite element techniques. There is a magnetic induction produced when a material is placed in a magnetic field of strength $\mathrm{H}$.

$$
\mathrm{B}=\mu_{O}(\mathrm{H}+\mathrm{M})
$$

Where $\mu_{o}$ is the permeability of free space and $M$ is the magnetic moment per unit, in term of volumetric magnetic susceptibility

$$
\begin{aligned}
\mathrm{M} & =\frac{m}{v} \\
\mathrm{M} & =\lambda \chi
\end{aligned}
$$

The magnetic field generated by a steady current $\mathrm{I}$ is described by the BIot-Savart Law

$$
\mathrm{B}=\frac{\mu_{O} \mathrm{I}}{4 \pi} \int \frac{\vec{r}}{r} d I
$$

The Ampere's law describes the fact an electric current can generate an induced magnetic field that is:

$$
\oint \mathrm{B} d \mathrm{I}=\mu_{O} \mathrm{I}_{\text {enc }}
$$

In a stable magnetic field, the integration along a magnetic loop is equal to the electric current the loop encloses

$$
\oint \mathrm{H} d \mathrm{I}=j
$$

The curl of a vector is given by

$$
\text { curl } \mathrm{A}=\nabla \times \mathrm{A}=\lim _{\nabla s \rightarrow 0} \frac{\oint \mathrm{A} d \mathrm{I}}{\nabla s} n
$$

Then we have:

$$
\operatorname{curl} \mathrm{H}=\nabla \times \mathrm{H}=\lim _{\nabla s \rightarrow 0} \frac{\oint \mathrm{H} d \mathrm{I}}{\nabla s} n=\frac{j}{\nabla_{S}}=J
$$

For a varying electric field with respect to time, we have:

$$
\nabla \times \mathrm{H}=J+\frac{\partial D}{\partial t}
$$

Faraday's law states that a moving magnet can generate an alternating electric field.

$$
\begin{gathered}
E=-\frac{\partial \Psi}{\partial t} \\
\nabla \times \mathrm{E}=-\nabla \mathrm{X} \frac{\partial \Psi}{\partial t}=-\frac{\partial}{\partial t}(\nabla \mathrm{X} \Psi) \\
\nabla \times \mathrm{E}=-\frac{\partial \mathrm{B}}{\partial t}
\end{gathered}
$$

Applying the Gaussian theorem which states that the integration of the divergence of a vector field over a certain volume is equivalent to the vector itself integrated over the entire closed surface that contains the volume

$$
\begin{gathered}
\int_{v} \nabla \cdot \mathrm{A} d v=\int_{s} \mathrm{~A} \cdot d s \\
D=\in \mathrm{E} \\
\mathrm{E}=\frac{q r}{4 \pi \in r^{3}}
\end{gathered}
$$


By applying the divergence theorem, we get:

$$
\begin{gathered}
\nabla \times D=\lim _{v \rightarrow 0} \int_{s} D d s=\lim _{v \rightarrow 0} \int_{s} \in \mathrm{E} d s=\lim _{v \rightarrow 0} \int_{s} \in \frac{q r}{4 \pi \in r^{3}} d s \\
\nabla \cdot D=\frac{q}{v}=\rho_{e} \\
\nabla \times \mathrm{H}=J+\frac{\partial D}{\partial t} \\
\nabla \times \mathrm{E}=-\frac{\partial \mathrm{B}}{\partial t} \\
\nabla \cdot \mathrm{B}=0 \\
\nabla \cdot D=\rho_{e}
\end{gathered}
$$

Finding the magnetic field everywhere around the hemozoin is: As the field oscillates in cyclic form, they cause the hémozoïne to spin around in a circular form. We can therefore calculate the magnetic induction inside the parasite. The cross-section area is given by:

$$
\pi a^{2}
$$

\section{a) AGNETIC INDUCTION}

Using the integral form of the Amper's law, we can find the magnetic induction

$$
\begin{gathered}
\oint \mathrm{B} \cdot d \overrightarrow{I_{1}}=\mu_{o} \int \overrightarrow{j n} d \alpha \\
\mathrm{B} \Phi \cdot 2 \pi \rho=\mu_{o} \int \frac{\mathrm{I}}{\mathrm{A}} d \alpha \\
\mathrm{B} \Phi \cdot 2 \pi \rho=\frac{\mu_{o} \mathrm{I}}{\mathrm{A}} \int d \alpha \\
\mathrm{B} \Phi \cdot 2 \pi \rho=\frac{\mu_{o} \mathrm{I}}{\mathrm{A}}[\alpha]_{b}^{a} \\
\mathrm{~B} \Phi \cdot 2 \pi \rho=\frac{\mu_{o} \mathrm{I}}{\mathrm{A}}\left(\pi \rho^{2}\right) \\
\mathrm{B} \Phi=\frac{\mu_{o} \mathrm{I} \rho}{2 \pi \alpha^{2}}
\end{gathered}
$$

The magnetization curve, as a function of the applied field, is a reverse S- shaped function. If the assumption is made that the hemozoin particles are identical implying that they have the same energy barrier same magnetic moment.

b) MAGNETIZATION OF THE HEMOZOIN PARTICLE by:

The expression of the magnetization of hemozoin is given

$$
\mathrm{M}(\mathrm{H})=n \mu \tanh \left(\frac{\mu_{o} \mathrm{H} \mu}{\mathrm{K}_{\mathrm{B}} \mathrm{T}}\right)
$$

Where $\mathrm{n}$ is the density of the hemozoin, $\mu_{\mathrm{o}}$ is the magnetic permeability of air, $\mu$ is the magnetic moment.

c) MAGNETIC SUSCEPTIBILITY OF HEMOZOIN

The magnetization in terms of susceptibility of hemozoin is:

$$
\mathrm{M}(\chi)=\chi \mathrm{H}
$$

$\chi$ is the magnetic susceptibility that can be expressed in complex form as:

$$
\chi=\chi^{\prime}+i \chi^{\prime \prime}
$$

d) MAGNETIC FIELD STRENGTH AND ITS AMPLITUDE

The magnetic field strength $\mathrm{H}$ of hemozoin has the amplitude as:

$$
\mathrm{H}(t)=\mathrm{H}_{o} \cos \omega t
$$

Where $\mathrm{H}_{\mathrm{o}}$ is the amplitude the magnetic field strength e) MAGNETIZATION IN TERM OF TIME

$$
\mathrm{M}(t)=\mathrm{H}_{o}\left(\chi^{\prime} \cos \omega t+\chi^{\prime \prime} \sin \omega t\right)
$$

\section{f) ENERGY PRODUCED DURING THE OXILLATING OF HEMOZOIN PARTICLES}

For collinear field in which the magnetization density is treated, the expression of energy is given by:

$$
d U=\mathrm{H} d \mathrm{~B}
$$

Replacing B by its expression, we have:

$$
d U=\mathrm{H} \cdot d\left[\mu_{o}(\mathrm{H}+\mathrm{M})\right]
$$

If we integrate the expression, we have:

$$
\Delta u=-\mu_{o} \oint \mathrm{M} d \mathrm{H}
$$

By replacing the expression of $\mathrm{M}$, we will get:

$$
\begin{gathered}
\Delta u=\mu_{o} \mathrm{H}_{o}{ }^{2} \int_{0}^{\frac{2 \pi}{\omega}}\left(\chi^{\prime} \cos \omega t+\chi \sin \omega t \chi^{\prime \prime}\right) \sin \omega t d t \\
\Delta u=2 \mu_{o} \mathrm{H}_{o} \int_{0}^{\frac{2 \pi}{\omega}} \sin ^{2} \omega t d t
\end{gathered}
$$

g) POWER DISSIPATED

The rotation of hemozoin particles results in Brownian mechanism of relaxation as well as the N'eel relaxation in which the magnetic moment rotates within the crystal of hemozoin. The volumetric power dissipation due to magnetic work done (energy) on a system in an adiabatic process is given by:

$$
p=f \Delta u=\mu_{o} \pi \chi^{\prime \prime} f \mathrm{H}_{o}^{2}
$$




\section{h) TEMPERATURE EXPRESSION}

The temperature expression is given by:

$$
\Delta \mathrm{T}=\frac{p \Delta t}{c}
$$

And the magnetic susceptibility function depending on frequency is:

$$
\chi^{\prime \prime}=\frac{\chi_{o} 2 \pi f \tau}{\left[1+(2 \pi f \tau)^{2}\right]}
$$

\section{Results and Discussion}

From the graph of Susceptibility in function of magnetic field strength (figure 3), we realize, the value of magnetic susceptibility of Hemozoin is increasing proportionally to the magnetic field strength, these show that the detection of hemozoin in an infected blood is possible due to cyclic effect of magnetic field on the nanoparticle. The electromagnetic properties of hemozoin are more excited when the field is applied constantly. That will enable detection of hemozoin for malaria diagnosis.

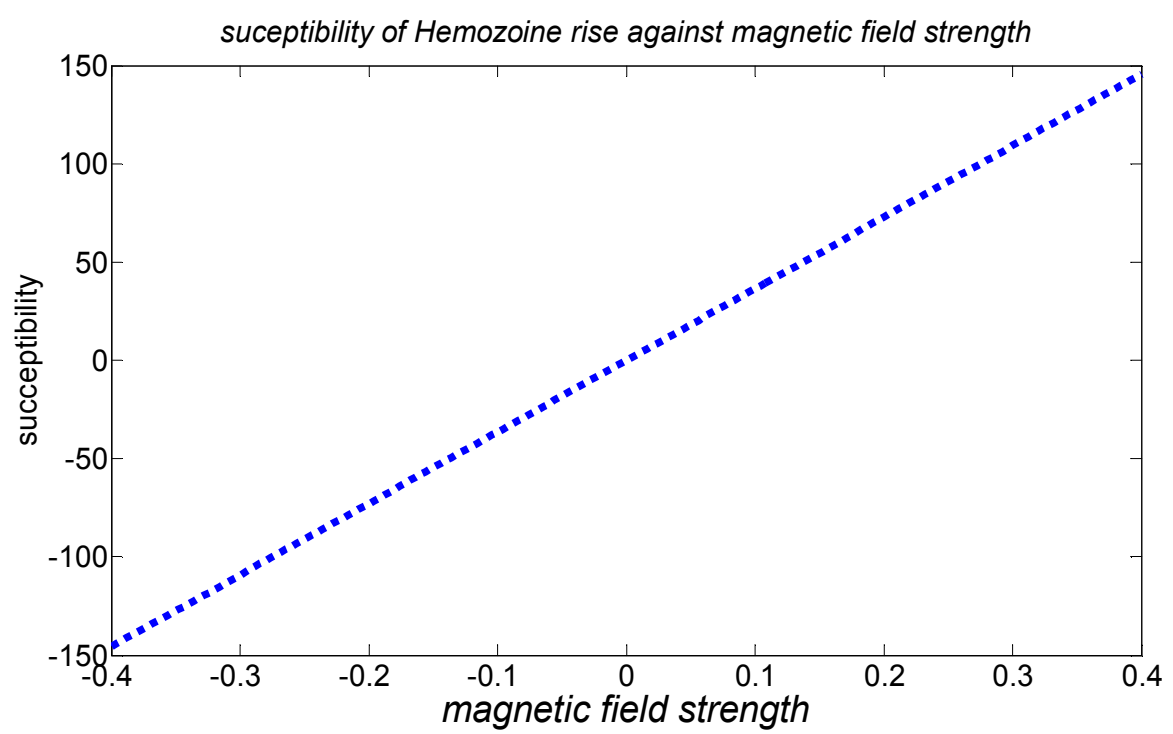

Figure 6. Excitation of hemozoin due to the presence of magnetic field.

But, the concentration of the super magnetic particles will cause the hemozoin to generate the heat.
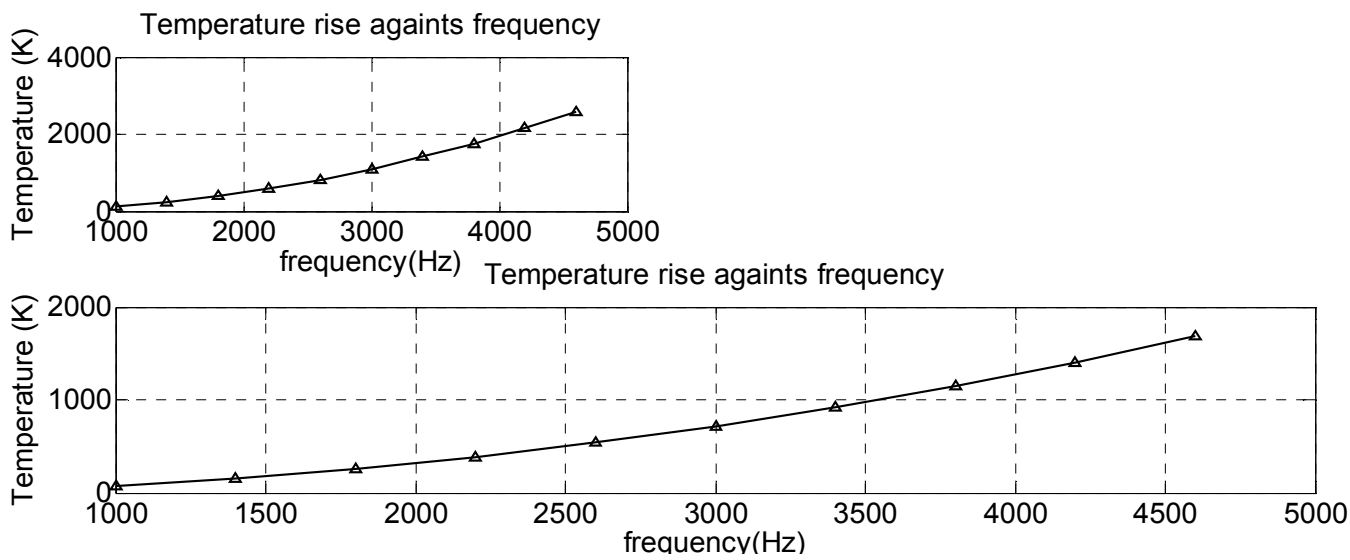

Figure 7. Temperature variation in term of frequency.

This curve presents the situation of hemozoin heat in heme, it shows how the temperature of hémozoïne increases in function of the frequencies at various values of field, and these are thanks to magnetic field for Nanoparticle excitation. We can see from the graph that at $3500 \mathrm{~Hz}$, the temperature of Hemozoin is $100 \mathrm{~K}$ (figure 4). The generation of heat will provide the energy because of the forces caused by the super particles in the heme.

The figure 8 is the evolution of the energy in the cell against the pulsation produced by the electromagnetic field. It is noticed on that graph that, more the magnetic field is applied to magnetize the particles of Hemozoin; the more heat is generated by the forces either by pull or push inside the blood. The energy occurred is increasing proportionally according to the values of pulsation. The pulsation value is due to the frequency of Hemozoin which is known by the different values of magnetic fields. 


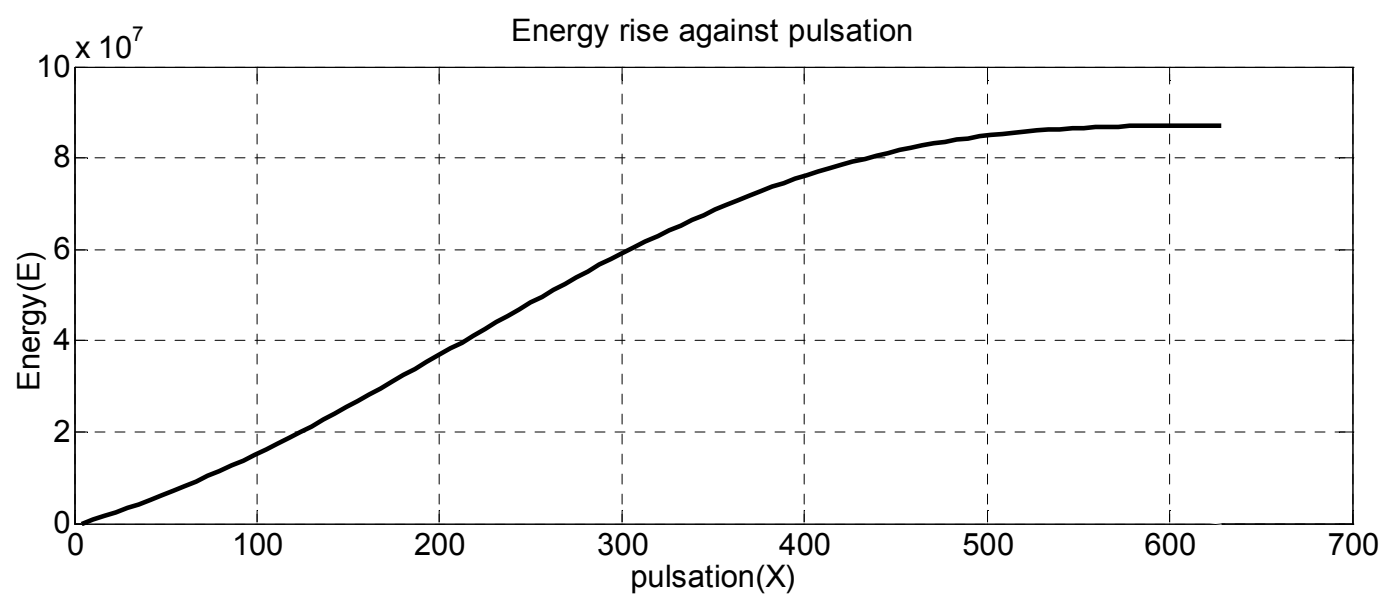

Figure 8. The energy against pulsation.

\section{Conclusion}

The objective of the work was model the effect of cyclic electromagnetic field on Hemozoin in infected blood for malaria diagnosis. The hemozoin in human cell have been idealized as magnetic nanoparticles that embedded in an infected blood. These are shown by its electromagnetic properties magnetic susceptibilities varying in terms of magnetic field applied. The potential effect of the cyclically activated magnetic field was modeled using a thermodynamic framework. Then at the end, generation and diffusion of heat due the presence of constant cyclically magnetic field have been determined. These to prove how the pull or push forces between the particles of Hemozoin are demonstrated by producing the heat. The energy inside the particle is also taken into consideration in the cell while doing the diagnosis of malaria.

\section{References}

[1] Wernig F. and Xu Q 2002, Mechanical stress -induced apoptosis in the cardiovascular systemprogress in Biological and Moluclar Biology, 78(2), pp. 105-137.

[2] Hayward, R.., Saliba, K. J., and Kirk, K. (2006) The pH of the digestive vacuole of theplasmodium falciparum is not associated with chlorquine resistance, J CellSci119, 1016-1025.

[3] Sullivan DJ (December 2002). "Theories on malarial pigment formation and quinolineaction". Int J Parasitol 32 (13): 164553.

[4] Yoed Rabin 2002, Cancer Treatment by Electromagnetic Activated Nanoheaters. Department of Mechanical Engineering.

[5] Robin D. Powell, Colonel Willam D. Tigertt, 1968, Drug resistance of parasite causing Human malaria.

[6] Solomon O. Abiola, 2012 Helmholtz Coil Design for NonInvasive Detection and Apoptosis of Breast Cancer and Malaria Parsites, via $\mathrm{Fe}_{2} \mathrm{O}_{3}, \mathrm{~F} \mathrm{e}_{3} \mathrm{O}_{4}$ Nanoparticles and Hemin.

[7] http://en.wikipedia.org/wiki/ Hysteresis, November 2017.
[8] https://www.google.com/ur source,October 2007.

[9] Q A Pankhurst, J Connolly, S K Jones and J Dobson, 2003 "Applications of magnetic nanoparticles in biomedicine".

[10] L. Rast and J. G. Harrison, 2010, "Computatiçonal Modeling of Electromagnetically Induced Heatinof Magnetic Nanoparticle Materials for Hyperthermic Cancer Treatment", Department of Physics, University of Alabama at Birmingham, Birmingham, Alabama 35294, USA.

[11] C. Phatak, R. Pokharel, M. Beleggia, M. De Graef, 2011, On the magnetostatics of chains of magnetic nanoparticles.

[12] Richard Fitzpatrick, 2008, Classical Electromagnetism: An intermediate level course

[13] Kannan M. Krishnan, 2016, Biomedical Nanomagnetics: A Spin through Possibilities in Imaging, Diagnostics, and Therapy.

[14] Henry C. Lai1 and Narendra P. Singh2016, Department of Bioengineering, University of Washington, Seattle, WA 98195-5061, USA.

[15] Olivia April 2011, Difference between oscillation and vibration

[16] A.-H. Lu, E. L. Salabas and F. Schüth, Angew. Chem., Int. Ed., $2007,46,1222-1244$.

[17] An-Hui Lu; E. L. Salabas, and Ferdi Schth (2007). Angew. Chem. Int. Ed. 46: 1222-1244.

[18] Wang X. et al, 2017, Application of Nanotechnology in Cancer Therapy and Imaging.

[19] Joerg Lehmann and Brita Lehmann 2016, Nanoparticle Thermotherapy: A New Approach in Cancer Therapy.

[20] KUMIKO SATU, January 2018 (AIST), A new diagnostic technology for malaria.

[21] Modélisation et simulation en épidémiologie G. Sallet INRIA \& IRD 2010.

[22] Geraldine TELLIER, septembre 2015, Etudes moléculaires et fonctionnelles de deux régulateurs de la rotéine phosphatase de type $1 \mathrm{chez}$ Plasmodium falciparum: I2 et eIF2 $\beta$.

[23] MN Wogu 2018, Evaluating Malaria prevalence using Clinical Diagnosis. 